\title{
28 Research Soure \\ Generation of Miniaturized Ovaries by in Vitro Culture From Mouse Gonads
}

\section{Si Won Jang}

Jeonbuk National University

Hoon Jang

Jeonbuk National University

Hyun Woo Choi ( $\nabla$ Choihw@jbnu.ac.kr)

Jeonbuk National University

\section{Research Article}

Keywords: Gonad, oogenesis, oocyte, in vitro, follicle formation

Posted Date: May 10th, 2021

DOl: https://doi.org/10.21203/rs.3.rs-478328/v1

License: (1) This work is licensed under a Creative Commons Attribution 4.0 International License.

Read Full License

Version of Record: A version of this preprint was published at Development \& Reproduction on September 1st, 2021. See the published version at https://doi.org/10.12717/DR.2020.25.3.173. 


\section{Abstract}

The incidence of infertility among individuals of reproductive age has been growing due to genetic and environmental factors, and considerable research efforts are focused on solving this issue. Ovarian development is an overly complex process in the body, involving the interaction between primordial germ cells and gonad somatic cells. However, follicles located in the center of the in vitro ovary are poorly formed or die owing to ovarian complexity, nutrient deficiency, and signaling deficiency. In the present study, we optimized methods for dissociating gonads and culture conditions for the in vitro generation of miniaturized ovaries. The gonads from embryos were dissociated into cell masses and cultured on a Transwell-COL membrane for 3 5 weeks. Approximately 12 follicles were present per in vitro ovary. We observed that miniaturized ovaries successfully matured to MII oocytes in vitro from 150 to $100 \mu \mathrm{m}$ gonad masses. This method will be useful for investigating follicle development and oocyte production.

\section{Introduction}

In reproductive and developmental biology, overcoming infertility remains a critical challenge. The development of egg production and conservation technology is crucial for successfully treating the growing number of patients presenting infertility. However, a considerable number of obstacles persist in the production of numerous functional oocytes. Therefore, to solve some of these challenges, research on in vitro oocyte generation from miniaturized ovaries is critical.

To date, scientists have investigated several strategies based on stem cell research to generate oocytes in vitro $^{1,2,3,4,5,6,7,8,9}$. For example, Hans and colleagues generated oocytes in vitro from embryonic stem cells (ESCs) ${ }^{11}$. Hayashi et al. have used a method to aggregate primordial germ cell (PGC)-like cells (PGCLCs) with germline somatic cells to produce oocytes ${ }^{12}$ and Obara and colleagues cultured 12.5 days post coitum (dpc) gonads and produced oocytes ${ }^{13}$. Moreover, oocytes were successfully generated using PGCs and germline somatic cells. The exploitation of an in vitro follicular development system allows the formation of simulated ovaries in the body through co-culture of PGCs and germline somatic cells, thus confirming oocyte formation and embryonic development. In vitro studies regarding ovarian formation techniques have been performed in various species, and research on human oocyte formation remains ongoing. Furthermore, differentiation of human oocytes has been attempted by differentiating induced pluripotent stem cells (iPSCs), which are human pluripotent stem cells, into human PGCs, and by heterogeneous co-culture of established human PGCs (hPGCs) and mouse germline somatic cells ${ }^{14,15}$. Although a few hundred follicles were obtained in a single ovary in vitro, the inner follicles did not receive adequate signals and were unable to fully develop, as distinct blood vessels were not developed like those seen in vivo. Thus, isolation of single follicles is necessary to stimulate follicle growth and maturation. These problems can be solved by establishing a miniaturized ovary capable of producing fewer follicles. By forming a miniaturized ovary, signals can reach the inner part, allowing nutritional supply. In addition, challenges in isolating individual follicles can be overcome, allowing a smooth transition to the in vitro maturation (IVM) process. Eventually, it will be easier to observe ovarian 
formation, and large amounts of oocytes can be efficiently obtained without issues such as oocyte loss or death.

Herein, we established a culture system producing a miniaturized ovary. First, we optimized the culture conditions for follicle formation from mouse gonads and then optimized the system for dissociation of gonads by employing suitable enzymes to generate miniaturized ovaries containing 5-30 follicles. In vitro oocytes from miniaturized ovaries could mature into MII oocytes.

\section{Methods}

\section{Reagents}

Pregnant female ICR mice at $12.5,13.5$, and $14.5 \mathrm{dpc}$ were employed to isolate gonad for dissociation experiments. The following reagents and chemicals were procured from respective suppliers: Dulbecco's modified Eagle's medium (1×) (DMEM; Gibco, 11965-092), phosphate-buffered saline (PBS; Gibco, cat. no. 10010-023), Minimum Essential Medium Eagle, alpha modification (1x) (a-MEM; Gibco, cat. no. 12571063 and 32571-036), 2-mercaptoethanol (55 mM; Gibco, cat. no. 21985-023), fetal bovine serum (FBS; Gibco, cat. no. 16000-044), Pen Strep Glutamine (100x) (Gibco, cat. no. 10378-016), ascorbic acid (150 mM; TCl, cat. no. G0394), ICl182 and 780 (Tocris, cat. no. 1047), follicle-stimulating hormone (FSH; Sigma, cat. no. F4021), epidermal growth factor (EGF; R\&D Systems, cat. no. 2028-EG-200), polyvinylpyrrolidone (PVP; Sigma, cat. no. PVP360-100G), human chorionic gonadotropin $(1,200 \mathrm{IU} / \mathrm{mL})$ (hCG; Sigma, cat. no. C8554), collagenase type IV (1 g/mL; Gibco, cat. no. 17104-019), trypsin-EDTA, 0.25\% (Gibco, cat. no. 25200-072), StemPro-34 SFM (1×), liquid (Gibco, cat. no. 10639-011), and hyaluronidase (TCl, cat. no. D8418).

\section{Equipment}

The following list of equipment was employed to perform the experiments: Clean bench (JSR, cat. no. JSCB-1500SB), water bath (JSR, cat. no. JSWB-22T), $\mathrm{CO}_{2}$ incubator (Astec, JP/SMA-165D), stereoscopic microscope (Olympus, cat. no. CKX53), a suction machine (Unim, cat. no. TFS-30), centrifuge (LABOGENE, cat. no. KLG122618050210), a hemocytometer (Paul Marienfeld, cat. no. 2960408), micropipettes (Eppendorf, cat. nos. EP3120000011, EP3120000020, EP3120000038, EP3120000046, EP3120000054, EP3120000062), sterile plastic tips ( $10 \mu \mathrm{L}, 200 \mu \mathrm{L}, 1,000 \mu \mathrm{L}$; Thermo Fisher), pipettors, sterile plastic pipettes $(5,10,25$, and $50 \mathrm{~mL}), 15$-mL conical centrifuge tubes (Thermo Fisher Scientific, cat. no. 338652 or equivalent), $50-\mathrm{mL}$ conical centrifuge tubes (Thermo Fisher Scientific, cat. no. 338652 or equivalent), four-well culture plates (Thermo Fisher Scientific, cat. no. 176740), Petri dish 35 mm (BDFalcon, cat. no. 353001), Petri dish 100 mm (BD-Falcon, cat. no. 353003), 24 well-tissue culture plates (Costar, cat. no. 3526), Transwell-COL membrane inserts (Costar, cat. no. 3496), sterile syringes (0.5 mL; BD, cat. no. 328821), tungsten wire (Nilaco, cat. no. W-461387), cell strainer ( $40 \mu \mathrm{m}, 70 \mu \mathrm{m}, 100 \mu \mathrm{m}, 150$ $\mu \mathrm{m}$; SPL, cat. nos. 93070, 93100), and $35 \mathrm{~mm}$ hexagon U-bottom plate (StemFIT 3D, cat. no. H2951000).

\section{DATA reporting}


No statistical methods were used to predetermine sample size. The experiments were not randomized. The investigators were not blinded to allocation during experiments and outcome assessment.

\section{Animal and Ethics statement}

All methods were performed in accordance with relevant guidelines and regulations of Jeonbuk National University. All experimental protocols were approved by the Institutional Animal Care and Use Committee of Jeonbuk National University (approval numbers CBNU-2019-00395). Pregnant female outbred albino ICR mice were purchased from Company of Samtako (Republic of Korea). Pregnant females were killed by cervical dislocation to obtain E12.5, E13.5 and E14.5 embryos. Only from female embryos, the gonads were isolated and then dissociated for generation of miniaturized ovaries. This study was carried out in compliance with the ARRIVE guidelines (http://www.nc3rs.org.uk/page.asp?id=1357).

\section{In vitro differentiation (IVDi) culture}

Female fetal gonads without mesonephros were cultured in Transwell-COL membranes (3.0- $\mu \mathrm{m}$ pore size, 24-mm diameter, Corning, Inc.) soaked in a-MEM-based IVDi medium (a-MEM supplemented with $2 \%$ FBS, $150 \mu \mathrm{M}$ ascorbic acid, $1 \times$ penicillin/streptomycin/L-glutamine, and $55 \mu \mathrm{M}$ 2-mercaptoethanol). From days 5 to 11 of culture, $10 \mu \mathrm{M}$ ICl 182780 was added to the a-MEM-based IVDi medium (henceforth, referred to as a-MEM). On day 4 of culture, the culture medium was changed to StemPro-based IVDi medium (StemPro-34 SFM supplemented with 10\% FBS, $150 \mu \mathrm{M}$ ascorbic acid, $1 \times$ penicillin/streptomycin/L-glutamine, and $55 \mu \mathrm{M}$ 2-mercaptoethanol). From days 7 to 10 of culture, 500 nM ICl182780 was added to the Stempro-34-based IVDi medium (henceforth, referred to as StemPro). On day 21 of culture, individual secondary follicles (2FLs) were manually dissociated using sharpened tungsten needles. Gonads were cultured for 21 days at $37^{\circ} \mathrm{C}$ under $5 \% \mathrm{CO}_{2}$ and $95 \%$ air. Approximately half of the medium in each well was replaced with fresh medium every other day.

\section{Isolation of 2FLs}

Electrically sharpened tungsten needles were used to isolate the individual $2 \mathrm{FLs}$. The interstitial cells between 2FLs were carefully removed. Then, 2FLs were separated from the ovaries and placed at regular intervals.

\section{In vitro growth (IVG) culture}

Single 2FLs on Transwell-COL membranes were soaked in IVG-a-MEM medium (a-MEM supplemented with $5 \% \mathrm{FBS}, 2 \%$ polyvinylpyrrolidone, $150 \mu \mathrm{M}$ ascorbic acid, $1 \times$ penicillin/streptomycin/L-glutamine, 100 $\mu \mathrm{M}$ 2-mercaptoethanol, $55 \mathrm{\mu g} \mathrm{ml}^{-1}$ sodium pyruvate, $0.1 \mathrm{IU} \mathrm{ml}^{-1} \mathrm{FSH}, 15 \mathrm{ng} \mathrm{ml}^{-1} \mathrm{BMP} 15$, and $15 \mathrm{ng} \mathrm{ml}^{-1}$ GDF9). After 2 days of culture, the medium containing BMP15 and GDF9 was withdrawn, and follicles were then incubated in $0.1 \%$ type IV collagenase. After washing several times with a-MEM supplemented with 5\% FBS, the follicles were cultured in IVG-a-MEM without BMP15 and GDF9. On day 11 of culture, cumulus-oocyte complexes grown on the membrane were picked using a fine glass capillary, cultured on 
Transwell-COL or Millicell membranes for another $9-13$ days at $37^{\circ} \mathrm{C}$ in medium, under $5 \% \mathrm{CO}_{2}$ and $95 \%$ air. Then, $1 \mathrm{~mL}$ of medium was added to the inside and outside of membrane inserts. Approximately half of the medium in each well was replaced with fresh medium every other day.

\section{IVM culture}

Cumulus-oocyte complexes were transferred to IVM medium (a-MEM supplemented with $5 \%$ FBS, $25 \mu \mathrm{g}$ $\mathrm{ml}^{-1}$ sodium pyruvate, $1 \times$ penicillin/streptomycin, $0.1 \mathrm{IU} \mathrm{ml} l^{-1} \mathrm{FSH}, 4 \mathrm{ng} \mathrm{ml}^{-1} \mathrm{EGF}$, and $1.2 \mathrm{IU} \mathrm{ml}^{-1} \mathrm{hCG}$ ). After $16 \mathrm{~h}$ of culture, swollen cumulus cells were stripped from oocytes by treatment with hyaluronidase, and then MII oocytes were determined by first polar body extrusion.

\section{Immunostaining}

In brief, oocytes were fixed for $4 \mathrm{~h}$ at $20^{\circ} \mathrm{C}$ in $4 \%$ paraformaldehyde. The cells were then washed with PBS and treated with PBS containing 10\% normal goat serum and $0.03 \%$ Triton X-100 for 45 min at room temperature. The primary antibodies used were anti-Ddx4 (polyclonal, 1:500; Abcam), anti-Oct3/4 (monoclonal, 1:500; Santa Cruz Biotechnology), and GDF9 (polyclonal, 1:500; Invitrogen). For detection of primary antibodies, fluorescently labeled (Alexa Fluor 488 or 568; Molecular Probes, Eugene, OR, USA) secondary antibodies were used according to the manufacturer's specifications. All immunofluorescence samples were analyzed using a confocal microscope (Zeiss LSM700).

\section{Statistical analysis}

All experiments were performed in triplicate, and generation of miniaturized ovaries using each method, the mean numbers of intact follicles per ovary was calculated by an arithmetical calculation.

\section{Results}

\section{Optimized culture conditions for follicle formation}

In our early experiments, female gonads obtained from E12.5 E14.5 mice were cultured in a-MEM or StemPro34 medium supplemented with $10 \%$ (vol/vol) FBS for 5 weeks on Transwell-COL membranes. First, we assessed the efficiency of follicle formation in the two culture media. Two control groups were prepared: one cultured using the a-MEM-based culture medium ${ }^{13}$ and the other cultured in StemPro34 medium $^{12}$. On employing the two cultures for preserving gonads, the formation of follicles was assessed, and secondary follicle formation was observed (Fig. 1a, b). Follicles generated using two different media gradually matured and produced oocytes (Fig. 1b). The in vitro oocytes expressed Oct4 and Ddx4, as well as the oocyte-specific marker GDF9 (Fig. 1c). Oct4 is expressed in the oocyte core, whereas Ddx4 is expressed on the oocyte $16,17,18,19,20,21$. These data revealed that, in both media, the cultures could form normal functional oocytes. Collectively, the gonads from the period between $12.5,13.5$, and $14.5 \mathrm{dpc}$ could develop into follicles in vitro on Transwell-COL membranes, irrespective of the medium employed. The follicle formation efficiency did not significantly differ between the two types of culture media. 
However, in vitro ovaries in StemPro medium contained considerably more follicles, demonstrating more rounded granulosa cells in the IVG medium than those in the a-MEM medium. Additionally, we confirmed that the granulosa cells were further expanded during the IVG process using StemPro-based media (Fig. 1d). Therefore, we used StemPro medium to perform all further experiments.

\section{Single-cell dissociation fails to form follicles}

Next, to determine whether a single follicle and oocyte possessing a three-dimensional (3D) structure could be formed, we cultured single cells dissociated from mice gonads at $12.5,13.5$, and $14.5 \mathrm{dpc}$ on a Transwell-COL dish using StemPro medium. Additionally, to confirm enzyme efficiency for gonad dissociation into single cells, two enzymes were assessed, i.e., trypsin-EDTA and collagenase IV. First, we used trypsin-EDTA to dissociate the gonads into single cells, which demonstrated high dissociation efficiency into single cells. We cultured single cells on Transwell-COL after trypsin-EDTA treatment to confirm dissociation of mice gonads at $12.5,13.5$, and $14.5 \mathrm{dpc}$. Spherical cells, which differed from the germline somatic cell shape, were found to grow on Transwell-COL membranes. We analyzed cultured globular cells, and expression levels of the germline cell marker Ddx4 were confirmed by immunocytochemistry (Fig. 2a). Spherical cells displayed Ddx4 expression on the cell surface; this was demonstrated by $12.5 \mathrm{dpc}$ gonads, as well as 13.5 and $14.5 \mathrm{dpc}$ gonads (Fig. 2a). Reportedly, gonadal PGCs are known to demonstrate oogonia, which can differentiate into meiotic cells through multiple cell

divisions during migration to the genital ridge ${ }^{22,23}$. These results demonstrated that, when single cells dissociated from gonads and PGCs and gonadal somatic cells were co-cultured in Transwell-COL, cocultured single cells could differentiate into oogonia-like cells expressing $\mathrm{Ddx} 4$, without follicle formation ${ }^{24}$.

Then, we aggregated and cultured PGCs and germline somatic cells on a U-bottom plate, as we failed to generate a 3D structure on Transwell-COL membranes. Single cells from gonads were seeded at a density of $2.5 \times 10^{5}$ per well in a hexagon-shaped U-bottom plate. The cells aggregated after 1 day, but no follicle formation was observed (Fig. 2b); these results were similarly observed for $12.5,13.5$, and $14.5 \mathrm{dpc}$ gonads. As another method for 3D culturing, we used Matrigel, which is widely used for organoid culture or 3D tissue culture $25,26,27$. Similar to previous results, it did not facilitate ovary formation (Fig. 2c). These results indicated that once the gonad is dissociated into a single cell, ovary formation is impossible even if clustered again.

\section{Method optimization for miniaturized ovary formation}

We employed several strategies to generate a minimally-sized ovary that can form a single follicle. Accordingly, the gonad was manually separated into three parts, and all three parts formed the ovary, producing fewer follicles than the control (Fig. 3a). In addition, follicles formed in this manner generated superior oocytes. Furthermore, the oocytes within the ovary were well-developed, similar to the oocytes on the outside. Signals and nutrients were well transmitted to the inner part of the ovary. However, the method failed to generate fewer ovarian follicles. Therefore, damage or loss of follicles can still occur 
during the isolation process. During this method, we used a thin syringe to mechanically cut the gonad into the smallest fragments possible. We then assessed ovary formation and observed that follicles were produced when gonads were cut to the minimum size without isolation into a single cell state. Accordingly, all gonads adequately formed ovaries; however, numerous follicles were generated. Moreover, the number of follicles appeared to random in the in vitro ovaries obtained (Fig. 3b). This method was unsuitable for generating uniform follicles in all ovaries, and it was difficult to individually isolate follicles. Based on these findings, it was confirmed that ovaries were formed even when the gonad was separated into a gonadal mass without isolation into a single cell. Next, we attempted to confirm whether ovary formation occurred when smaller gonadal masses were achieved using a collagenase IV suspension rather than mechanical separation. Using the collagenase IV enzyme, we observed that gonads were dissociated into an appropriate gonad mass that could form ovaries. In the present study, the efficiency of dissociation into an appropriate gonadal mass was increased by controlling the collagenase IV treatment time and suspension. Following collagenase IV treatment for more than 2 min, gonadal masses were broken into single cells; when treatment was performed for less than 2 min, ovaries producing numerous follicles were formed. After approximately 2 min of collagenase IV treatment in suspension, a few minimally crushed masses could be identified. We cultured the masses and found that ovaries with a fewer number of follicles were formed. The ovaries generated using this method were smaller in size than those generated using the previous methods; however, a random number of follicles were produced (Fig. 3c). This strategy successfully reduced ovary size and follicle number; however, precise standardization remained challenging.

Finally, we cultured gonad tissues after breaking them down to a uniform size using $150 \mu \mathrm{m}, 100 \mu \mathrm{m}, 70$ $\mu \mathrm{m}$, and $40 \mu \mathrm{m}$ meshes. Collagenase VI treatment was performed for $2 \mathrm{~min}$, followed by repeated suspension two or three times; in the absence of adequate collagenase treatment, all gonads will be similar in size as control gonads. In order to determine the minimum mass size that can accurately form follicles, the gonad was cultured by dividing it into four groups after passing through $150 \mu \mathrm{m}, 100 \mu \mathrm{m}, 70$ $\mu \mathrm{m}$, and $40 \mu \mathrm{m}$ meshes. Thus, gonad masses were cultured in four groups of 150-100 $\mu \mathrm{m}, 100-70 \mu \mathrm{m}$, 70-40 $\mu \mathrm{m}$, and $40 \mu \mathrm{m}$ or less (Fig. $3 \mathrm{~d}$ ). When cultured by breaking the gonad using a $40 \mu \mathrm{m}$ mesh, the gonadal mass formed ovaries but no follicles (Fig. 3e). Additionally, the gonadal mass passed through the $70 \mu \mathrm{m}$ mesh failed to generate follicles (Fig. 3e). In contrast, the gonadal mass passed through the $100 \mu \mathrm{m}$ mesh and retained in the $70 \mu \mathrm{m}$ mesh only formed four ovaries, which produced follicles (Fig. 3f). However, owing to the low ovarian production rate, it was challenging to produce numerous oocytes. Gonadal mass passed through the $150 \mu \mathrm{m}$ mesh and retained in the $100 \mu \mathrm{m}$ mesh was equal or slightly larger than $100 \mu \mathrm{m}$ sized mass, formed miniaturized ovaries, and contained an average of 12 uniform follicles per ovary (Fig. 3f). In gonadal masses ranging between $150 \mu \mathrm{m}$ and $100 \mu \mathrm{m}$ in size, ovaries generated normally developed follicles during IVG (Fig. 4a). These results indicate that a minimum gonadal mass of $100 \mu \mathrm{m}$ is required to form an ovary capable of generating follicles. In other words, when the gonad is crushed to a size less than $100 \mu \mathrm{m}$, the cells are damaged, and ovaries cannot be formed. In this experiment, we used collagenase IV treatment, manual cutting, and passage through a mesh. On using a mesh, we were able to identify the most efficient and suitable size. Undoubtedly, the 
method was less time-consuming, resulted in reduced cell loss and damage during isolation, and produced ovaries with fewer follicles. Although there are technical limitations to generating ovaries that form single follicles, these issues can be surpassed. Ten days after IVG, oocytes were well-developed into germinal vesicle (GV) oocytes (Fig. 4a). GV oocytes were treated with IVM medium containing FSH, hCG, and EGF for $16 \mathrm{~h}$ at $37^{\circ} \mathrm{C}$. This medium was used to resume meiosis. After $16 \mathrm{~h}$, the oocytes extruded the first polar body (Fig. 4b).

\section{Discussion}

The generation of a large number of in vitro oocytes is a critical step in overcoming infertility. Therefore, developing the most efficient and appropriate method to produce numerous in vitro oocytes remains crucial in reproductive biology and developmental biology. To date, the generated ovaries have produced hundreds of follicles, such that the inner follicles fail to generate normal oocytes owing to a lack of signal, nutrient factors, and lack of space for cell growth ${ }^{12,13}$. Accordingly, it is important to isolate several follicles generated in the ovary. This leads to cell loss or cell damage, which results in a lower percentage of GV oocytes from the ovaries. Culturing several ovaries that produce fewer follicles in vitro would be less time-consuming and less complex, increasing the production of GV oocytes. Moreover, it will be easier to observe ovary formation in vitro. Thus, it will be possible to overcome the infertility problem by efficiently obtaining numerous oocytes.

In the present study, we compared the efficiency of cultures with methods used to form fewer follicles. We compared only a-MEM media and a-MEM with StemPro34 to determine which media would be ideal. There was no noticeable difference between cultures using the two media; however, StemPro34 appeared to generate a small number of follicles with more rounded oocytes. The gonads cultured under these conditions formed a normal ovary, well-produced oocytes. These oocytes expressed Oct4 (pluripotent marker), Ddx4 (germ cell marker), and GDF9 (oocyte-specific marker). Notably, both cultures must be initiated with a-MEM media. The gonads are composed of PGCs and somatic cells. We first devised a method to break the gonads into single cells on a Transwell-COL membrane. We employed $0.25 \%$ trypsinEDTA, which produced a more significant number of intact integrins and faster dissemination ${ }^{28}$. Single cells that were completely broken following trypsin treatment failed to form ovaries, generating only oogonia-like cells. Next, when single cells were aggregated on the U-bottom plate, we assessed whether they could form normal ovaries. Hayashi and colleagues isolated PGCs into single cells and then aggregated them with germline somatic cells to successfully form a simulated ovary ${ }^{12}$. They used PGCs, differentiated from ESCs, and these cells appeared to be similar to the PGC stage of E 9.5 in vivo. In contrast, PGCs used in this study were isolated from the germline 12 days after implantation. Additional investigations are needed to elucidate the developmental process of PGCs and the effect of single-cell dissociation. Single cells from the $12.5,13.5$, and $14.5 \mathrm{dpc}$ gonads could be aggregated into Transwell$\mathrm{COL}$ or U-bottom plates; however, follicle formation could not be achieved. These cells formed spheres rather than ovaries. Spherical cells were examined for $D d x 4$ expression. Based on immunochemistry, surface Ddx4 expression was confirmed. The ovary was not formed in the single cell state, with 
differentiation into oogonia-like cells observed ${ }^{23,29}$. In several species, germ cells are connected by cytoplasmic bridges and can share cytoplasmic contents between germ cells at different stages of development until a mature gamete is formed. All germ nuclei share a common cytoplasm- this is not an ideal condition for effective transcriptional control ${ }^{30,31,32,33,34,35}$. Pepling and spradling analysis in mouse ovaries showed the presence of intercellular bridges (ring canals) between the ovarian germ cells at E11.5 and $\mathrm{E} 17.5^{34}$. For ovary formation, it can be inferred that the cytoplasmic bridges between germ cells are not destroyed when a gonad mass of at least $100 \mu \mathrm{m}$ is formed.

In conclusion, we observed that PGCs with somatic cells could differentiate into oogonia-like cells when dissociated into single cells using gonadal somatic cells. Furthermore, we revealed that when gonads were dissociated into single cells, ovary formation could not be achieved following aggregation of PGC and gonad somatic cells in the U-bottom plate.

Several methods have been used to form ovaries with a small number of oocytes. We attempted to manually cut the gonadal tissues into three equal parts. In this method, the number of oocytes from the ovaries was lower than that of the control. However, prior to the IVG process, it appears that signals are not well transmitted to the inner part of the ovary; hence, inner oocytes fail to develop normally. Next, we manually cut the gonads as feasible using a syringe. This method generated ovaries that formed fewer follicles, which was undoubtedly more effective than the previous method. However, there were difficulties in achieving a uniform number of follicles, resulting in a considerable number of follicles. We then employed collagenase type IV, frequently used for tissue dissociation. Following treatment with collagenase IV, the cells were less damaged and broke down into an appropriate gonadal mass. The most appropriate treatment time was determined by adjusting the collagenase IV treatment time; the gonadal mass was incompletely broken when treated for approximately $2 \mathrm{~min}$. Therefore, we determined that treatment with collagenase minimizes cell damage and is more efficient than trypsin treatment. Herein, we observed that prolonged incubation and suspension in collagenase IV could result in cell damage and a failure to produce ovaries. We dissociated the gonadal mass considering only the collagenase IV treatment time and suspension. The number of oocytes formed per in vitro ovary was random, and the size tended to vary. However, the ovaries formed few follicles. Hence, we developed a more efficient method to evenly generate a small number of follicles. Previously, Kim and colleagues generated ovaries using a mesh to isolate follicles from ovarian tissues; they optimized the system for follicle isolation from ovarian tissue using diameters of $0.254 \mathrm{~mm}$ and $380 \mu \mathrm{m}$ meshes $^{36}$. We attempted to generate miniaturized ovaries in vitro, and dissociated gonads were sequentially passed through $150 \mu \mathrm{m}, 100 \mu \mathrm{m}$, $70 \mu \mathrm{m}$, and $40 \mu \mathrm{m}$ meshes followed by seeding on Transwell-COL membranes. Accordingly, we observed that ovaries were formed only with the 150-100 $\mu \mathrm{m}$ and 100-70 $\mu \mathrm{m}$ masses, with no ovaries observed in groups below $70 \mu \mathrm{m}$. In the 100-70 $\mu \mathrm{m}$ group, except for 4 ovaries, most did not form ovaries. Thus, the probability of reproduction was low. In the 150-100 $\mu \mathrm{m}$ group, approximately 12 follicles were formed on average, and the follicles were well-developed following even the distribution of appropriate signals and nutrients. 
Our in vitro system will be useful for developing an ovary model to visualize and overcome cell loss, time consumption, and cell damage issues encountered during the isolation process. Therefore, our system can generate more well-developed oocytes for fertilization. This method would help solve infertility challenges for all potential parents and will significantly impact national fertility.

\section{Declarations}

\section{Acknowledgements.}

This work was supported by the National Research Foundation of Korea (NRF) grant funded by the Korea government (MSIP; Ministry of Science, ICT \& Future Planning) (NRF-2017R1C1B5077043).

\section{Author information}

Department of Agricultural Convergence Technology, Jeonbuk National University, Jeonju, 54896, Republic of Korea

Si Won Jang and Hyun Woo Choi

Department of life Science, Jeon-buk National University, Jeonju city, Republic of Korea

Hoon Jang

Department of Animal Science, JeonBuk National University, Jeonju, 54896, Republic of Korea

Hyun Woo Choi

Contribution.

S.W.J. performed the experiments. S.W.J. and H.W.C. wrote the manuscript. H.J. performed and supervised statistical analyses. H.W.C. designed and supervised the study and corrected the manuscript. All authors read and approved the final version of the manuscript.

\section{Competing interests}

The author(s) declare no competing interests.

\section{References}

1 Evans, M. J. \& Kaufman, M. H. Establishment in culture of pluripotential cells from mouse embryos. Nature292, 154-156, doi:10.1038/292154a0 (1981).

2 Takahashi, K. et al. Induction of pluripotent stem cells from adult human fibroblasts by defined factors. Cel/131, 861-872, doi:10.1016/j.cell.2007.11.019 (2007). 
3 Takahashi, K. \& Yamanaka, S. Induction of pluripotent stem cells from mouse embryonic and adult fibroblast cultures by defined factors. Cel/126, 663-676, doi:10.1016/j.cell.2006.07.024 (2006).

4 Hayashi, K., Ohta, H., Kurimoto, K., Aramaki, S. \& Saitou, M. Reconstitution of the mouse germ cell specification pathway in culture by pluripotent stem cells. Cel/146, 519-532, doi:10.1016/j.cell.2011.06.052 (2011).

5 Hayashi, K. et al. Offspring from oocytes derived from in vitro primordial germ cell-like cells in mice. Science338, 971-975, doi:10.1126/science.1226889 (2012).

6 Aramaki, S. et al. A Mesodermal Factor, T, Specifies Mouse Germ Cell Fate by Directly Activating Germline Determinants. Developmental Cel/27, 516-529, doi:https://doi.org/10.1016/j.devcel.2013.11.001 (2013).

7 Kurimoto, K. et al. Quantitative Dynamics of Chromatin Remodeling during Germ Cell Specification from Mouse Embryonic Stem Cells. Cell stem cel/16, 517-532, doi:https://doi.org/10.1016/j.stem.2015.03.002 (2015).

8 Murakami, K. et al. NANOG alone induces germ cells in primed epiblast in vitro by activation of enhancers. Nature529, 403-407, doi:10.1038/nature16480 (2016).

9 Nakaki, F. et al. Induction of mouse germ-cell fate by transcription factors in vitro. Nature501, 222-226, doi:10.1038/nature12417 (2013).

10 Thomson, J. A. et al. Embryonic stem cell lines derived from human blastocysts. Science282, 11451147, doi:10.1126/science.282.5391.1145 (1998).

11 Hubner, K. et al. Derivation of oocytes from mouse embryonic stem cells. Science300, 1251-1256, doi:10.1126/science.1083452 (2003).

12 Hikabe, O. et al. Reconstitution in vitro of the entire cycle of the mouse female germ line. Nature539, 299-303, doi:10.1038/nature20104 (2016).

13 Morohaku, K. et al. Complete in vitro generation of fertile oocytes from mouse primordial germ cells. Proc Natl Acad Sci U S A113, 9021-9026, doi:10.1073/pnas.1603817113 (2016).

14 Irie, N. et al. SOX17 Is a Critical Specifier of Human Primordial Germ Cell Fate. Cel/160, 253-268, doi:https://doi.org/10.1016/j.cell.2014.12.013 (2015).

15 Sasaki, K. et al. Robust In Vitro Induction of Human Germ Cell Fate from Pluripotent Stem Cells. Cell stem cel/17, 178-194, doi:https://doi.org/10.1016/j.stem.2015.06.014 (2015).

16 Noce, T., Okamoto-Ito, S. \& Tsunekawa, N. Vasa Homolog Genes in Mammalian Germ Cell Development. Cell Structure and Function26, 131-136, doi:10.1247/csf.26.131 (2001). 
17 Saitou, M., Barton, S. C. \& Surani, M. A. A molecular programme for the specification of germ cell fate in mice. Nature418, 293-300, doi:10.1038/nature00927 (2002).

18 Tanaka, S. S. et al. Regulation of expression of mouse interferon-induced transmembrane protein like gene-3, Ifitm3 (mil-1, fragilis), in germ cells. Developmental dynamics : an official publication of the American Association of Anatomists230, 651-659, doi:10.1002/dvdy.20085 (2004).

19 Okamura, D., Tokitake, Y., Niwa, H. \& Matsui, Y. Requirement of Oct3/4 function for germ cell specification. Developmental biology317, 576-584, doi:10.1016/j.ydbio.2008.03.002 (2008).

20 Sabour, D. et al. Identification of genes specific to mouse primordial germ cells through dynamic global gene expression. Human molecular genetics20, 115-125, doi:10.1093/hmg/ddq450 (2011).

21 Pisarska, M. D., Barlow, G. \& Kuo, F. T. Minireview: roles of the forkhead transcription factor FOXL2 in granulosa cell biology and pathology. Endocrinology152, 1199-1208, doi:10.1210/en.2010-1041 (2011).

22 Hayashi, K. \& Saitou, M. Generation of eggs from mouse embryonic stem cells and induced pluripotent stem cells. Nature protocols8, 1513-1524, doi:10.1038/nprot.2013.090 (2013).

23 Saitou, M. \& Miyauchi, H. Gametogenesis from Pluripotent Stem Cells. Cell stem cel/18, 721-735, doi:10.1016/j.stem.2016.05.001 (2016).

24 Lei, L. et al. Stage-specific germ-somatic cell interaction directs the primordial folliculogenesis in mouse fetal ovaries. Journal of cellular physiology208, 640-647, doi:10.1002/jcp.20702 (2006).

25 Lang, S. H. et al. Experimental prostate epithelial morphogenesis in response to stroma and threedimensional matrigel culture. Cell Growth Differ12, 631-640 (2001).

26 Pierzchalska, M., Grabacka, M., Michalik, M., Zyla, K. \& Pierzchalski, P. Prostaglandin E2 supports growth of chicken embryo intestinal organoids in Matrigel matrix. Biotechniques52, 307-315, doi:10.2144/0000113851 (2012).

27 Dolega, M. E., Abeille, F., Picollet-D'hahan, N. \& Gidrol, X. Controlled 3D culture in Matrigel microbeads to analyze clonal acinar development. Biomaterials52, 347-357, doi:10.1016/j.biomaterials.2015.02.042 (2015).

28 Brown, M. A. et al. The use of mild trypsinization conditions in the detachment of endothelial cells to promote subsequent endothelialization on synthetic surfaces. Biomaterials28, 3928-3935, doi:10.1016/j.biomaterials.2007.05.009 (2007).

29 Hickford, D. E., Frankenberg, S., Pask, A. J., Shaw, G. \& Renfree, M. B. DDX4 (VASA) Is Conserved in Germ Cell Development in Marsupials and Monotremes1. Biology of Reproduction85, 733-743, doi:10.1095/biolreprod.111.091629 \%J Biology of Reproduction (2011). 
30 Pushpa, K., Kumar, G. A. \& Subramaniam, K. Translational Control of Germ Cell Decisions. Results Probl Cell Differ59, 175-200, doi:10.1007/978-3-319-44820-6_6 (2017).

31 Fawcett, D. W. Intercellular bridges. Experimental Cell Research8, 174-187, doi:https://doi.org/10.1016/0014-4827(61)90347-0 (1961).

32 Gondos, B. Germ cell degeneration and intercellular bridges in the human fetal ovary. Zeitschrift für Zellforschung und Mikroskopische Anatomie138, 23-30, doi:10.1007/BF00307075 (1973).

33 Gondos, B. \& Conner, L. A. Ultrastructure of developing germ cells in the fetal rabbit testis. The American journal of anatomy136, 23-42, doi:10.1002/aja.1001360104 (1973).

34 Pepling, M. E., de Cuevas, M. \& Spradling, A. C. Germline cysts: a conserved phase of germ cell development? Trends in Cell Biology9, 257-262, doi:https://doi.org/10.1016/S0962-8924(99)01594-9 (1999).

35 Ventelä, S. in Cell-Cell Channels (eds Frantisek Baluska, Dieter Volkmann, \& Peter W. Barlow) 208-216 (Springer New York, 2006).

$36 \mathrm{Kim}, \mathrm{E}$. J. et al. Comparison of Follicle Isolation Methods for Mouse Ovarian Follicle Culture In Vitro. Reproductive sciences (Thousand Oaks, Calif.)25, 1270-1278, doi:10.1177/1933719117737851 (2018).

\section{Figures}

\section{Figure 1.}

A

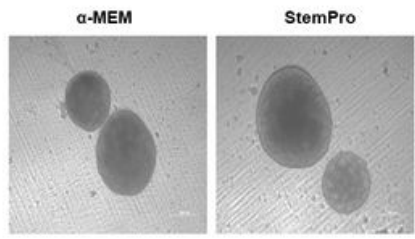

C

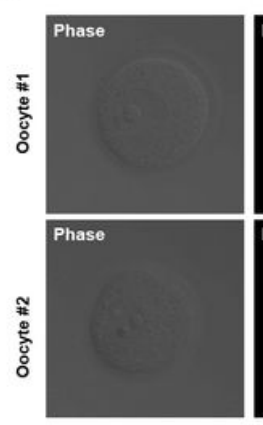

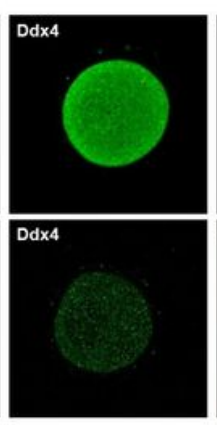

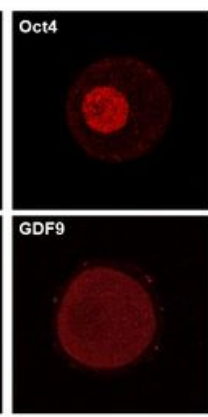

B
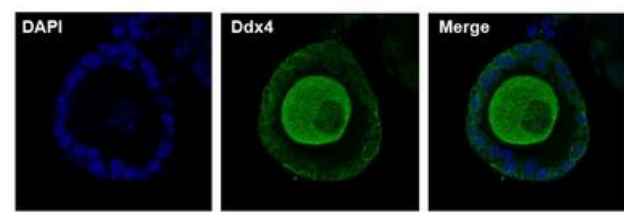

D

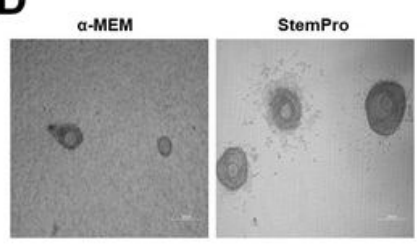


Figure 1

Optimized culture condition for oocyte formation A. Ovary culture. Representative images of ovaries at two weeks of culture in the a-MEM-based medium and StemPro-based medium (scale bar, $200 \mu \mathrm{m}$ ) B. Immunostaining results for the follicular expression of Ddx4 in vitro $\mathrm{C}$. Oocyte phase and expression of the Ddx4, Oct4, and oocyte-specific marker GDF9 in vitro D. Image of the follicular growth between the aMEM-based medium and StemPro-based medium (scale bar, $200 \mu \mathrm{m}$ )

\section{Figure 2.}

A

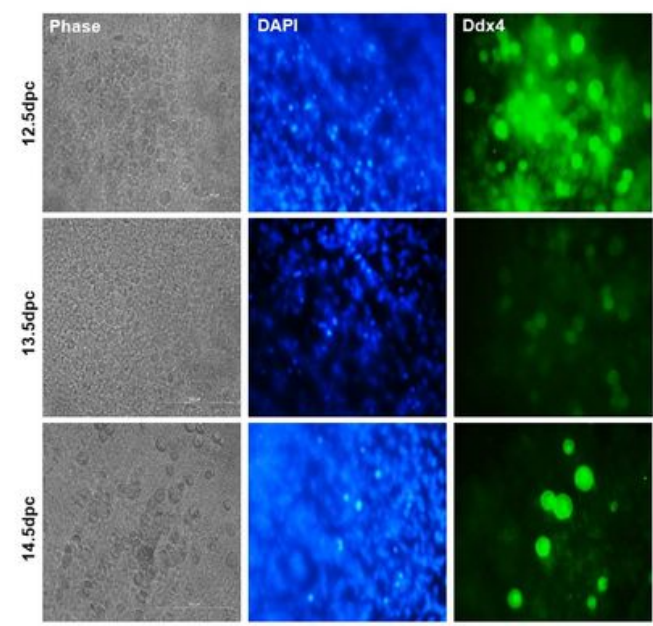

B

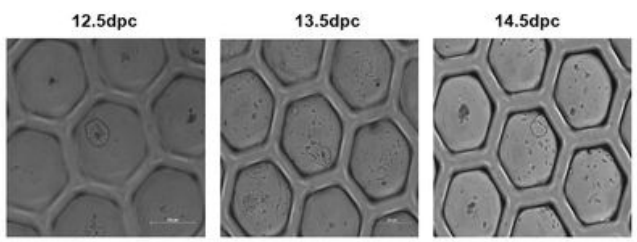

C

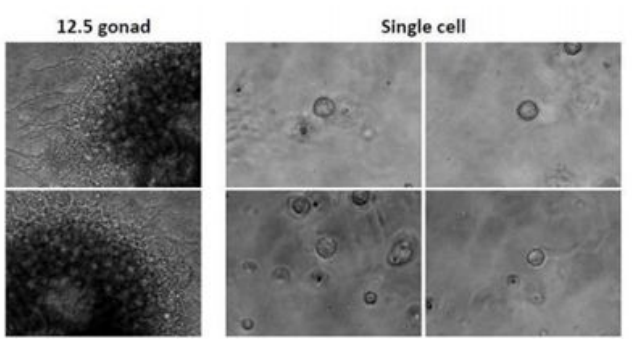

\section{Figure 2}

Single-cell dissociation fails to form follicles A. Phase and immunostaining data for expression of Ddx4 in oogonia-like cells developed 12.5, 13.5, and 14.5 days post coitum (dpc) (scale bar, $200 \mu \mathrm{m}$ ) B. Follicle is not formed in the U-bottom plate (scale bar, $200 \mu \mathrm{m}$ ) C. Cultivation of gonad cells in Matrigel. 
Figure 3.

A

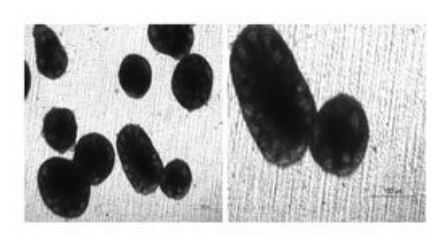

B

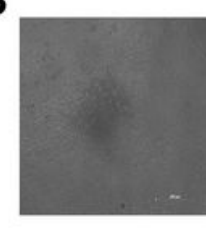

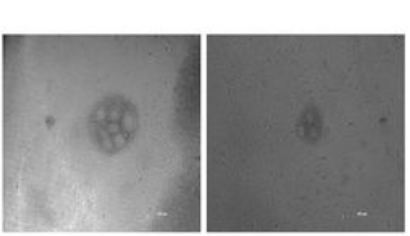

E
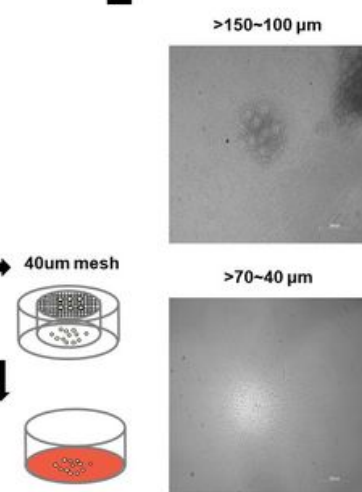

C

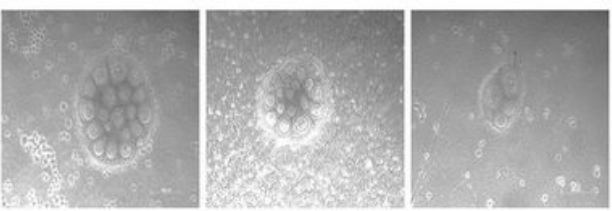

$\mathbf{F}$

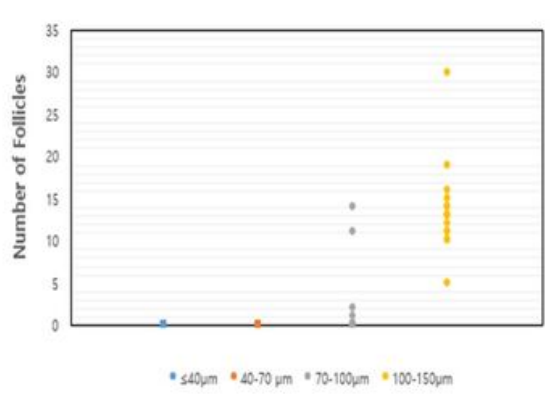

\section{Figure 3}

Method optimization for generation of miniaturized follicles per ovary A. Image of the ovaries from gonadal tissues dissociated into three parts (left scale bars, $40 \mu \mathrm{m}$; right scale bar, $100 \mu \mathrm{m}$ ) B. Image of the ovaries from gonadal tissues dissociated by mechanical cutting (scale bar, $200 \mu \mathrm{m}$ ) C. Image of the

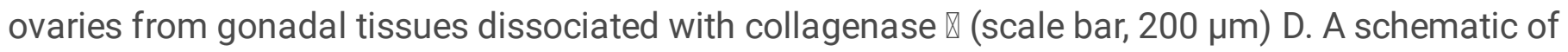
miniaturized ovary production in vitro $\mathrm{E}$. Image of the ovaries from gonadal tissues dissociated using $150,100,70$, and $40 \mu \mathrm{m}$ meshes (scale bar, $200 \mu \mathrm{m}$ ) F. Number of follicles in miniaturized ovaries generated from different gonadal mass sizes $(n=16$, Experiments were performed in triplicate). 
Figure 4.

A
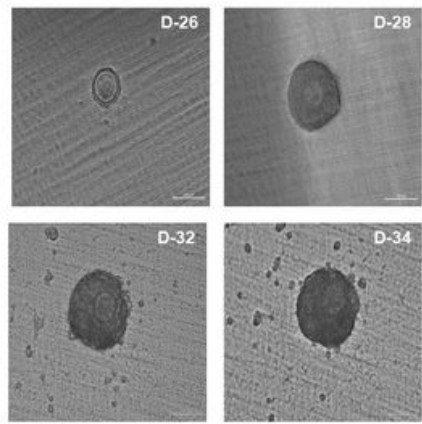

B

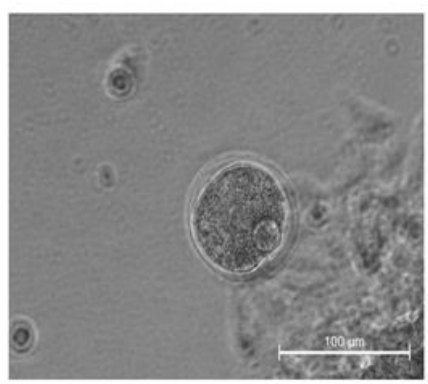

\section{Figure 4}

Oocyte maturation from the miniaturized ovary A. Representative image of the follicle cultivated on a Transwell-COL membrane on days 26-35 (scale bar, $100 \mu \mathrm{m}$ ) B. Image of the first polar body in MII oocyte (scale bar, $100 \mu \mathrm{m}$ ) 